\title{
Spin Relaxation Processes in Quantum Well Structures of $\mathrm{Cd}_{1-x} \mathrm{Mn}_{x} \mathrm{Te} / \mathrm{Cd}_{1-x} \mathrm{Mg}_{x} \mathrm{Te}$
}

\author{
A. Khachapuridze, V.Yu. Ivanov, M. Godlewski \\ AND G. KARCZEWSKI \\ Institute of Physics, Polish Academy of Sciences \\ al. Lotników 32/46, 02-668 Warsaw, Poland
}

\begin{abstract}
Fast spin relaxation of $\mathrm{Mn}^{2+}$ ions in a magnetic quantum well of CdMnTe with $1 \%$ Mn fraction is related to a very efficient spin-flip interaction between $\mathrm{Mn}$ ions and free carriers. This mechanism of spin relaxation becomes dominant at increased excitation densities. The observed response of the photoluminescence bands to the $\mathrm{Mn}^{2+}$ magnetic resonance indicates that free carriers are heated at the magnetic resonance conditions. A decrease in formation/recombination rates of free and trion excitons is observed. Donor bound exciton photoluminescence is enhanced, which we relate to delocalization of free excitons, caused by interaction with microwave heated free carriers.
\end{abstract}

PACS numbers: 71.35.Ji, 72.25.Rb, 76.70.Hb, 78.55.Et

\section{Introduction}

Processes of energy transfer from inequilibrium carriers to magnetic ions were studied by several authors [1-10]. The studies were performed for various diluted magnetic semiconductor (DMS) systems containing $\mathrm{Mn}^{2+}$ ions. It was concluded that hot carriers relax their excess energy by fast spin-flip relaxation with $\mathrm{Mn}^{2+}$ ions, which results in an increase in $\mathrm{Mn}^{2+}$ effective spin temperature. It was also concluded that such spin-flip processes are faster than spin-lattice relaxation processes [3], even though the latter can be enhanced for DMS with an increased Mn fraction. Such enhancement of spin-lattice relaxation rate was explained by a diffusion of a spin excitation to regions of an increased Mn concentration [11]. Even in these cases spin-lattice relaxation is too slow to account for dynamics of spin-related processes, e.g., for dynamics of magnetic polaron formation [12]. 
Much less of attention was paid to spin relaxation processes of magnetic ions. These relaxation processes proceed in most of the cases by inefficient spin-lattice interaction, mediated by acoustic phonons (see e.g. [3]). This is why spin-flip carrier-Mn interactions can be dominant for very diluted samples, for which spin-lattice relaxation is fairly inefficient [11]. Such possibility was already indicated by our previous optically detected magnetic resonance (ODMR) investigations [6, 13]. If spin relaxation of $\mathrm{Mn}^{2+}$ ions is dominated by $\mathrm{Mn}$-to-free carriers spin-flip interactions, gas of free carriers can be heated at the magnetic resonance conditions [6]. The latter can be observed in the ODMR as a change in intensity of relevant photoluminescence (PL) emission bands $[6,13]$. In this work we looked for such responses of the PL spectra to magnetic resonance conditions. The ODMR study was performed to evaluate the efficiency of Mn-to-free carriers spin-flip interactions, by studying the ODMR signals and their spectral responses for a low dimensional DMS system of CdMnTe/CdMgTe.

\section{Experimental}

ODMR and electron spin resonance (ESR) investigations were performed on either a $Q$-band $(36 \mathrm{GHz}$ ) or $60 \mathrm{GHz}$ microwave system developed by the authors, with a microwave cavity mounted in a split-coil magnet of the Oxford Instruments, using either argon laser or semiconductor laser diode $(1.92 \mathrm{eV})$ for the PL excitation. In the ODMR experiment we measured the PL changes (a change of an intensity or a shift of the PL spectral position) at magnetic resonance conditions, which were measured synchronously with on-off modulated microwave power. The experiments were performed on a $\mathrm{CdMnTe} / \mathrm{CdMgTe}$ single quantum well (QW) structure with 1\% Mn fraction in the magnetic QW and with 20\% of $\mathrm{Mg}$ in the CdMgTe barriers. The structure was grown by molecular beam epitaxy on a GaAs substrate covered with a thick CdTe buffer layer.

\section{Experimental results and discussion}

In the ODMR we observed a strong signal with Lande $g$-factor of 2 , which we attribute, based on a separate ESR study, to the $\mathrm{Mn}^{2+}$ magnetic resonance. The ODMR signal was detected via a change in intensity and/or in a spectral position of the relevant QW PL emissions. The magnitude of the signal and sign (decrease or increase in PL) depended on a microwave power used, on an excitation density, and on PL detection energy.

In this paper we concentrate on discussion of spectral responses of the PL bands to ODMR signals, by measuring the so-called ODMR-PL spectra. In the ODMR-PL we measured modifications of the PL bands at the magnetic resonance conditions. In particular, we studied the dependence of the ODMR-PL spectra on an excitation density, using the above band gap excitation. The excitation 
intensity was varied between 0.01 and $15 \mathrm{~W} / \mathrm{cm}^{2}$, i.e., we used relatively low excitation densities to avoid lattice heating.

In Fig. 1 we show the response of the excitonic QW PLs to the magnetic resonance ( $Q$-band data), measured at the lowest excitation density. At such excitation density the PL spectrum is dominated by a free exciton transition (X PL), which decreases in intensity and shifts up-in-energy at the magnetic resonance. X PL shifts by about $2 \mathrm{meV}$, when we applied a microwave power at the $\mathrm{Mn}^{2+}$ magnetic resonance conditions. Such up-in-energy shift of the X PL indicates a significant decrease in the sample magnetization at the magnetic resonance conditions, as was already reported in the early ODMR investigations [13, 14]. The resulting suppression of a Zeeman splitting (magnetization) is large and at a microwave power of about $200 \mathrm{~mW}$ can even reach $60 \%$. The shift and decrease in intensity of the X PL is accompanied by the appearance of a weak PL at the low energy wing of the X PL in the ODMR-PL spectrum. This new PL is due to a donor bound exciton (DBE) PL. The appearance of this PL indicates some delocalization of $\mathrm{X}$ excitons, as will be explained later on.



Fig. 1. Response of the QW PL emissions to the $\mathrm{Mn}^{2+}$ magnetic resonance conditions, observed for the lowest excitation density used in our study.

To account for the macroscopic decrease in the sample magnetization, observed in the ODMR, we must assume that $T_{1}$ spin relaxation for $\mathrm{Mn}^{2+}$ ions is slow, at low temperature and at low excitation density. We verified this statement by measuring dynamics of magnetic resonance-induced PL changes, using a time-resolved ODMR experiment. The time dependence of the signals was measured under pulse of microwave power, with a time resolution of about 20 ns. The relevant result is shown in Fig. 2. We measured a decay of the X PL signal observed at a high energy upon the microwave pulse applied at the magnetic resonance conditions, and an increase in the low energy X PL peak after turning off the microwaves. Both processes are characterized by the same characteristic time of $10 \mu \mathrm{s}$, which relates to a spin relaxation time in our system. This value 
of the spin relaxation time is characteristic of the spin-lattice interaction in bulk CdMnTe with Mn fraction of $1 \%$ [11, 12]. We thus verified that spin relaxation is fairly slow at low excitation densities and that this relaxation is dominated by the spin-lattice interaction.

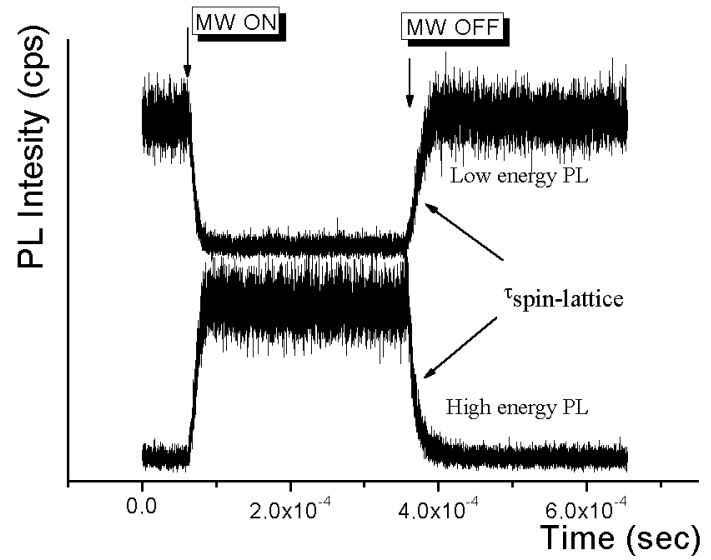

Fig. 2. Dynamics of the microwave induced (microwave pulse at the $\mathrm{Mn}^{2+}$ magnetic resonance conditions) modifications of the X PL, by setting detection at high energy X PL (at magnetic resonance conditions) and low energy X PL (observed at a turned off microwave power).

A distinctly different PL and ODMR-PL spectra are observed at high excitation densities, as is shown in Fig. 3 for the largest excitation density used by us. An additional PL band, attributed to a radiative decay of negatively charged excitons (trions, labeled as $\mathrm{X}^{-} \mathrm{PL}$ ), is observed together with $\mathrm{X}$ and DBE PL emissions. The three PL bands do not change their spectral positions and only change their intensities at the magnetic resonance conditions. The $\mathrm{X}$ and $\mathrm{X}^{-} \mathrm{PLs}$ are reduced in intensity, whereas the DBE PL is enhanced once microwave power is turned on (Fig. 3).

A similar PL response we observed setting conditions for the electron cyclotron resonance $(\mathrm{CR})$ in the optically detected cyclotron resonance (ODCR, see [15] for explanation of the technique). The $\mathrm{X}$ and $\mathrm{X}^{-}$PLs are reduced in intensity and the DBE PL is enhanced once we heat free electrons at the CR conditions. We also observed small up-in-energy shift of the X PL, by a localization energy, which indicates delocalization of excitons by interactions with hot carriers. The ODCR investigations indicate thus that we must delocalize excitons to enhance the DBE formation in CdTe or CdMnTe QWs [16]. The fact that we observe identical PL responses in the ODMR (at high excitation densities) and ODCR indicates that free carriers are heated at the magnetic resonance conditions, similarly to the situation in the ODCR. Scattering of hot carriers (heated 


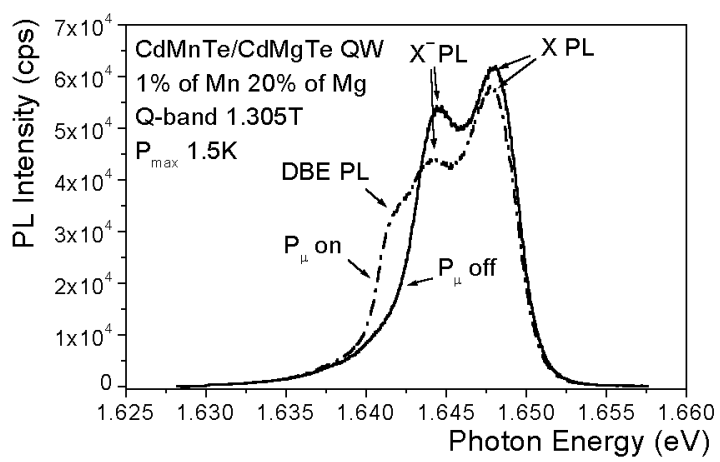

Fig. 3. Response of the QW PL emissions to the $\mathrm{Mn}^{2+}$ magnetic resonance conditions, observed for the largest excitation density used in our study.

at either magnetic resonance or $\mathrm{CR}$ conditions) on excitons may result in their delocalization and/or dissociation, observed as changes of the PL intensities. This explains the ODMR-PL spectra observed by us at high excitation densities.

Changes in intensity of excitonic PL emissions (see Fig. 3) are not accompanied by spectral shifts of their positions. The shift gradually disappears with increasing excitation density and is very small for density of $15 \mathrm{~W} / \mathrm{cm}^{2}$. We thus conclude that spin-excited $\mathrm{Mn}^{2+}$ ions evidently recombine much faster at increased excitation densities, i.e., in the presence of free carriers. The magnitude of shortening of spin relaxation time, observed at increased excitation densities, could not be estimated by us, which was due to a limited time resolution of our ODMR system. From a separate time-resolved PL experiment, performed for a similar system, we concluded that spin relaxation time must be indeed very fast [17].

Concluding, our ODMR investigations indicate a high efficiency of $\mathrm{Mn}^{2+}$ spin relaxation by spin-flip interactions with free carriers, which become dominant at increased excitation densities. We expect that a high efficiency of such spin-flip interactions explains the observed puzzling shortening of the $\mathrm{Mn}^{2+}$ intra-shall PL decay time, observed for several DMS systems [17].

This work was partly supported by the grant No. 5 P03B 00720 of the State Committee for Scientific Research granted for years 2001-2003.

\section{References}

[1] Yu.G. Semenov, Sov. Phys. JETP 54, 794 (1981).

[2] S.M. Ryabchenko, Yu.G. Semenov, O.V. Terletskii, Sov. Phys. JETP 55, 557 (1982).

[3] H. Krenn, W. Zawadzki, G. Bauer, Phys. Rev. Lett. 55, 1510 (1985).

[4] D.D. Awschalom, J. Warnock, S. von Molnar, Phys. Rev. Lett. 58, 812 (1987).

[5] H. Krenn, K. Kaltenegger, T. Dietl, J. Spałek, G. Bauer, Phys. Rev. B 39, 10918 (1989). 
[6] M. Godlewski, R.R. Gałązka, I. Tsimperidis, T. Gregorkiewicz, C.A.J. Ammaerlaan, Acta Phys. Pol. A 87, 177 (1995).

[7] V.D. Kulakovskii, M.G. Tyazhlov, A.I. Filin, D.R. Yakovlev, A. Waag, G. Landwehr, Phys. Rev. B 54, R8333 (1996).

[8] M.G. Tyazhlov, A.I. Filin, A.V. Larionov, V.D. Kulakovskii, D.R. Yakovlev, A. Waag, G. Landwehr, Sov. Phys. JETP 85, 784 (1997).

[9] M.G. Tyazhlov, V.D. Kulakovskii, A.I. Filin, D.R. Yakovlev, A. Waag, G. Landwehr, Phys. Rev. B 59, 2050 (1999).

[10] B. König, I.A. Merkulov, D.R. Yakovlev, W. Ossau, S.M. Ryabchenko, M. Kutrowski, T. Wojtowicz, G. Karczewski, J. Kossut, Phys. Rev. B 61, 16870 (2000).

[11] T. Strutz, A.M. Witowski, P. Wyder, Phys. Rev. Lett. 68, 3912 (1992).

[12] T. Dietl, P. Peyla, W. Grieshaber, Y. Merle d'Aubigne, Phys. Rev. Lett. 74, 474 (1995).

[13] S.J.C.H.M. van Gisbergen, M. Godlewski, R.R. Gałązka, T. Gregorkiewicz, C.A.J. Ammerlaan, Nguyen The Khoi, Phys. Rev. B 48, 11767 (1993).

[14] A.V. Komarov, S.M. Ryabchenko, O.V. Terletskii, I.I. Zheru, R.D. Ivanchuk, Sov. Phys. JETP 46, 318 (1977).

[15] M. Godlewski, W.M. Chen, B. Monemar, CRC Crit. Rev. Solid State Mater. Sci. 19, 241 (1994).

[16] M. Godlewski, Opt. Appl. 30, 463 (2000).

[17] M. Godlewski, V.Yu. Ivanov, A. Khachapuridze, S. Yatsunenko, Phys. Status Solidi $B$ 229, 533 (2002). 